\title{
Quantum efficiency and oscillator strength of site-controlled InAs quantum dots
}

Albert, F.; Stobbe, Søren; Schneider, C.; Heindel, T.; Reitzenstein, Stephan; Höfling, S.; Lodahl, Peter; Worschech, L.; Forchel, A.

Published in:

Applied Physics Letters

Link to article, DOI:

$10.1063 / 1.3393988$

Publication date:

2010

Document Version

Publisher's PDF, also known as Version of record

Link back to DTU Orbit

Citation (APA):

Albert, F., Stobbe, S., Schneider, C., Heindel, T., Reitzenstein, S., Höfling, S., Lodahl, P., Worschech, L., \& Forchel, A. (2010). Quantum efficiency and oscillator strength of site-controlled InAs quantum dots. Applied Physics Letters, 96(15), 151102. https://doi.org/10.1063/1.3393988

\section{General rights}

Copyright and moral rights for the publications made accessible in the public portal are retained by the authors and/or other copyright owners and it is a condition of accessing publications that users recognise and abide by the legal requirements associated with these rights.

- Users may download and print one copy of any publication from the public portal for the purpose of private study or research.

- You may not further distribute the material or use it for any profit-making activity or commercial gain

- You may freely distribute the URL identifying the publication in the public portal 


\title{
Quantum efficiency and oscillator strength of site-controlled InAs quantum dots
}

\author{
F. Albert, ${ }^{1}$ S. Stobbe ${ }^{2}$ C. Schneider,${ }^{1}$ T. Heindel, ${ }^{1}$ S. Reitzenstein, ${ }^{1, a)}$ S. Höfling, ${ }^{1}$ \\ P. Lodahl, ${ }^{2}$ L. Worschech, ${ }^{1}$ and A. Forchel ${ }^{1}$ \\ ${ }^{1}$ Technische Physik, Wilhelm Conrad Röntgen Research Center for Complex Material Systems, \\ Universität Würzburg, Am Hubland, D-97074 Würzburg, Germany \\ ${ }^{2}$ Department of Photonics Engineering, DTU Fotonik, Technical University of Denmark, Ørsteds Plads 343, \\ DK-2800 Kongens Lyngby, Denmark
}

(Received 19 December 2009; accepted 22 March 2010; published online 12 April 2010)

\begin{abstract}
We report on time-resolved photoluminescence spectroscopy to determine the oscillator strength $(\mathrm{OS})$ and the quantum efficiency $(\mathrm{QE})$ of site-controlled InAs quantum dots nucleating on patterned nanoholes. These two quantities are determined by measurements on site-controlled quantum dot (SCQD) samples with varying thickness of the capping layer. We determine radiative and nonradiative decay rates, from which we calculate an OS of 10.1 \pm 2.6 and an encouragingly high $\mathrm{QE}$ of $(47 \pm 14) \%$ for the SCQDs. The nonideal QE is attributed to nonradiative recombination at the etched nanohole interface. (C) 2010 American Institute of Physics. [doi:10.1063/1.3393988]
\end{abstract}

Semiconductor quantum dots (QDs) are fascinating nanoscopic structures for photonics and future information technology. Due to enormous research activities devoted to these high-quality nanostructures tremendous progress has been achieved in their growth, their physical understanding, and their integration into low mode volume microcavities. The related QD-microcavity systems are of particular interest for cavity quantum electrodynamics (cQED) studies, ${ }^{1,2}$ and devices like single-photon sources ${ }^{3}$ and ultralow-threshold QD lasers. ${ }^{4,5}$ However, the random position of self-organized QDs hinders deterministic cQED-experiments and presents a severe problem for large scale device fabrication. A possible solution of this issue lies in the growth and device integration of site-controlled quantum dots (SCQDs). In recent years, significant progress has been achieved in this field. For instance, periodic arrays of QDs with well-defined positions have been realized ${ }^{6-10}$ and integrated into microcavities. ${ }^{11-13}$ SCQDs technology typically combines top-down and bottom-up nanotechnology techniques. Our approach relies on etched nanoholes acting as seeds for the self-organized formation of the QDs. ${ }^{10,14}$ While a well-controlled growth of SCQDs is realized in this way, it is also known that the etching of the nanoholes introduces charged defect states in the vicinity of the QDs. These defect states have detrimental effect on the quality of the QDs and lead, for instance, to large single-exciton linewidths, which typically exceed $100 \mu \mathrm{eV} .^{13,15}$ Considerable efforts have been undertaken to reduce and understand the enhanced emission linewidth of SCQDs, which is mainly attributed to spectral diffusion. ${ }^{14,16,17}$ However, a detailed characterization of their quality in terms of the quantum efficiency $(\mathrm{QE})$ and the oscillator strength (OS), i.e., properties that are of particular importance for the study of cQED effects and their application in future light sources, is still missing.

In this paper we apply a method recently developed by Johansen et al. ${ }^{18,19}$ to determine the QE and the OS of InAs SCQDs by time-resolved spectroscopy. The method builds on the work by Drexhage, who demonstrated a modified de-

${ }^{\text {a)} E l e c t r o n i c ~ m a i l: ~ s t e p h a n . r e i t z e n s t e i n @ p h y s i k . u n i-w u e r z b u r g . d e . ~}$ cay rate for fluorescing molecules close to a reflecting surface. ${ }^{20}$ The radiative decay rate is modified because the proximity of the interface leads to a characteristic oscillation of the local density of optical states (LDOS) at the position of the QDs when varying the thickness of the capping layer. As a result, the radiative decay rate of the QDs shows also an oscillatory variation as a function of the distance $z$ to the interface while the nonradiative decay is not affected by a change in the LDOS, which allows one to extract the nonradiative and radiative decay rates of the QDs separately from which the QE and the OS of the QDs can be determined.

The InAs SCQDs were grown by solid source molecular beam epitaxy on a GaAs (100) wafer. The sample growth starts with a distributed Bragg reflector (DBR) consisting of six pairs of quarter wavelength thick GaAs and AlAs layers, which reflects the emission from the SCQDs and ensures enhanced intensity in photoluminescence (PL) experiments. The DBR is followed by a $228 \mathrm{~nm}$ thick GaAs buffer layer. 13 nanohole arrays with a pitch of $1 \mu \mathrm{m}$ and a lateral extension of $200 \times 200 \mu \mathrm{m}^{2}$ are processed ex situ by means of electron beam lithography, wet chemical etching, and a subsequent cleaning step. ${ }^{13}$ The growth continues with an $8 \mathrm{~nm}$ thick GaAs buffer layer before two stacked layers of InAs, which are separated by a $10 \mathrm{~nm}$ thick barrier consisting of 5 $\mathrm{nm} \mathrm{GaAs}, 3 \mathrm{~nm} \mathrm{Al} \mathrm{l}_{0.34} \mathrm{Ga}_{0.66} \mathrm{As}$, and $2 \mathrm{~nm} \mathrm{GaAs}$, are grown on the sample. The initial InAs layer does not contain optically active QDs but previous studies showed its importance for the formation of the SCQDs in the second InAs layer. ${ }^{13}$ The inset of Fig. 2 shows a scanning electron microscopy (SEM) image of a reference sample. One clearly observes a well-ordered array of SCQDs in the second layer and no signature of QDs at interstitial sites. Finally, a $283 \mathrm{~nm}$ thick GaAs capping layer is deposited on the sample. Afterwards 13 SCQD samples with capping layers between 37 and $283 \mathrm{~nm}$ were realized by wet chemical etching. The actual thickness of the capping layer was determined for each subsample with an error of $\pm 8 \mathrm{~nm}$. A cross sectional SEM image and a schematic view of a sample with $z=283 \mathrm{~nm}$ are depicted in Fig. 1 . 


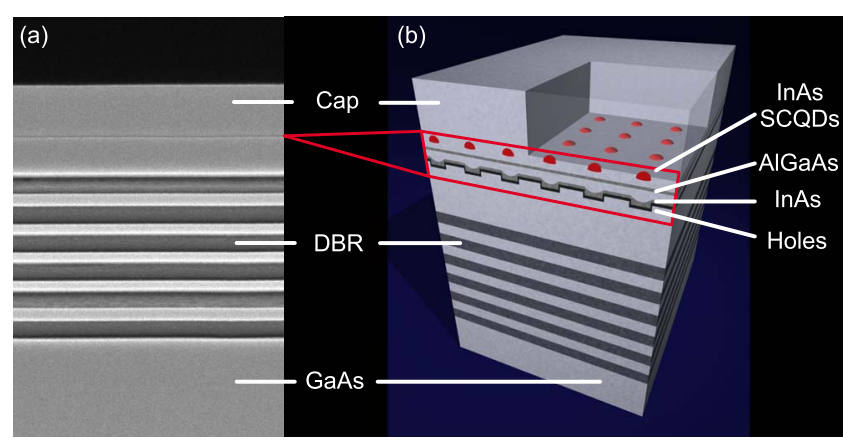

FIG. 1. (Color online) (a) SEM image of the cleaved edge of the sample with a distance of $283 \mathrm{~nm}$ between the SCQDs and the GaAs-air interface. (b) Schematic view of the sample. Optically active SCQDs are located in the second InAs layer.

To begin with we recorded time-integrated PL of the ensemble consisting of a few hundred SCQDs. The SCQDs were excited by a mode-locked Ti:sapphire laser emitting $150 \mathrm{fs}$ wide pulses at a repetition rate of $82 \mathrm{MHz}$ and a wavelength of $780 \mathrm{~nm}$. The PL setup has a spectral resolution of $150 \mu \mathrm{eV}$ and a temporal resolution of 100 ps. Figure 2 shows a PL spectrum of the sample with a distance of $283 \mathrm{~nm}$ between the SCQDs and the GaAs-air interface. Similar to standard self-assembled QDs, an inhomogeneously broadened emission band centered at $930 \mathrm{~nm}$ is observed. In addition, a micro-photoluminescence $(\mu \mathrm{PL})$ emission spectrum from a single SCQD line is presented in Fig. 2. The SCQD exhibits a full width at half maximum of $610 \mu \mathrm{eV}$ which is in agreement with previous studies on SCQDs. Compared to standard self-assembled QDs with emission linewidths below $10 \mu \mathrm{eV},{ }^{21}$ the significantly broader linewidth of the SCQDs is attributed to spectral diffusion due to charge traps at the etched layer of the nanoholes. ${ }^{14}$ As a consequence, important information about the homogeneous linewidth and, thus, information about the radiative decay rate of SCQDs cannot be assessed by simple PL measurements.

To obtain insight into the optical quality of the SCQDs we studied their decay characteristics under variation in $z$ [cf. Fig. 3(b)] for three different wavelengths (920, 930, and 940 $\mathrm{nm}$ ) within the inhomogeneously broadened emission spectrum. Figure 3(a) shows characteristic PL decay curves from SCQDs placed at two different distances $(z=72$ and $169 \mathrm{~nm})$

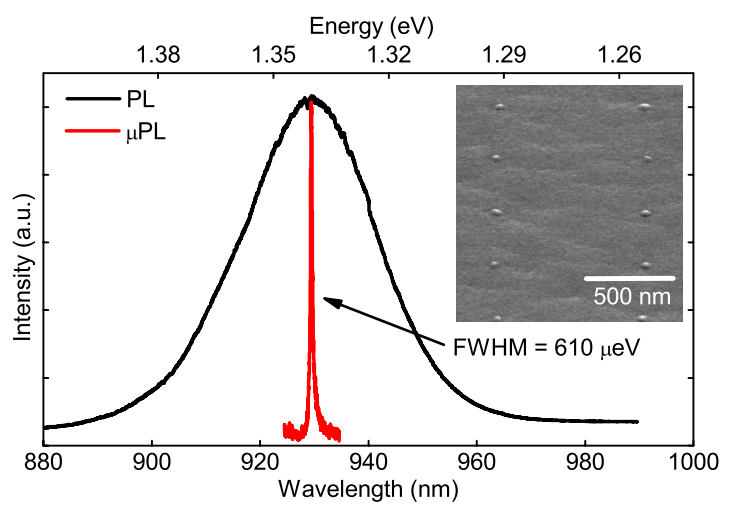

FIG. 2. (Color online) PL spectra of the SCQD sample with a distance of $283 \mathrm{~nm}$ between the SCQDs and the GaAs-air interface recorded at $T$ $=10 \mathrm{~K}$. The inset shows an SEM image of an uncapped reference sample with well-ordered arrays of SCQDs (pitch: $1 \mu \mathrm{m}$ ), which was tilted for better visibility.
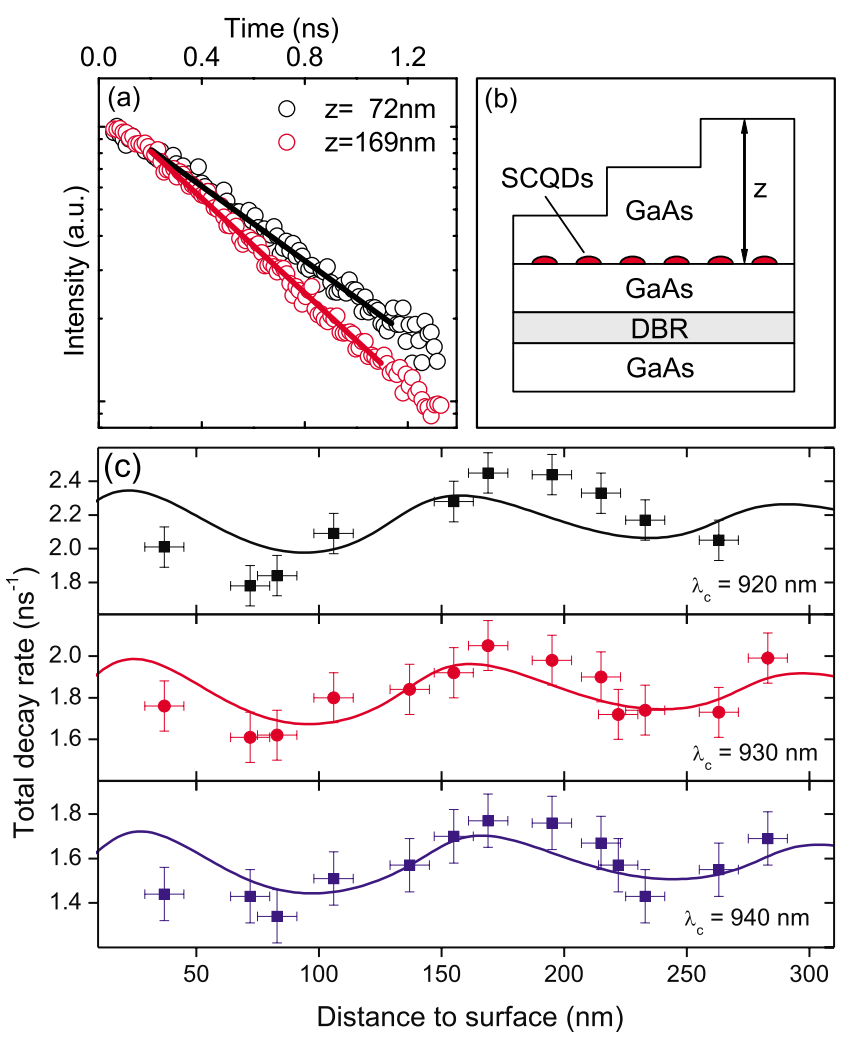

FIG. 3. (Color online) (a) Semilogarithmic plot of the PL decay curves for SCQDs positioned at $72 \mathrm{~nm}$ (black, upper curve) and $169 \mathrm{~nm}$ (red/gray, lower curve) distance to the GaAs-air interface recorded at $\lambda_{c}=930 \mathrm{~nm}$. (b) Schematic illustration of the sample. (c) Experimental decay rates (symbols) and calculated LDOS (solid lines) as a function of distance $z$ to the surface for $\lambda_{c}=920,930$, and $940 \mathrm{~nm}$.

to the GaAs-air interface on a semilogarithmic scale [detection window: $(930 \pm 2) \mathrm{nm}]$. By fitting the data at short delay times with an exponential decay function we extract the recombination rate of bright excitons ${ }^{18}$ (solid black and solid red/gray line) giving total decay rates of $1.61 \mathrm{~ns}^{-1}$ for $z=72 \mathrm{~nm}$ and $2.05 \mathrm{~ns}^{-1}$ for $z=169 \mathrm{~nm}$. The different decay rates reflect the change in the LDOS associated with a different thickness of the capping layer. The decay rates of all samples are extracted in the same way from time-resolved measurements and are summarized in Fig. 3(c) as a function of $z$. A clear oscillatory behavior is observed for all traces that correspond to detection at the wavelengths $\lambda_{c}=920,930$, and $940 \mathrm{~nm}$.

In order to facilitate a quantitative evaluation of the $\mathrm{QE}$ and OS we have compared the measured decay rates to the LDOS following the procedure outlined in Refs. 18 and 19. The measured decay rate $\Gamma(\omega, z)$ of a QD can be expressed by

$$
\Gamma(\omega, z)=\Gamma_{\text {nrad }}(\omega)+\Gamma_{\text {rad }}^{\mathrm{hom}}(\omega) \frac{\rho(\omega, z)}{\rho_{\text {hom }}(\omega)},
$$

where $\Gamma_{\text {nrad }}(\omega)$ denotes the nonradiative decay rate and $\Gamma_{\text {rad }}^{\text {hom }}(\omega)$ represents the radiative decay in a homogeneous medium with an LDOS $\rho_{\text {hom }}(\omega)$ at the frequency $\omega$ of the optical transition. The second term in Eq. (1) corresponds to the radiative decay rate which depends via the projected LDOS $\rho(\omega, z)$ on $z$. In the present case the DBR stack and the $\mathrm{Al}_{0.34} \mathrm{Ga}_{0.66} \mathrm{As}$ layer below the SCQD layer modify the LDOS significantly and must be taken into account. We have 
included all layers in the heterostructure by introducing generalized Fresnel coefficients, which are calculated by a transmission matrix method. The result of this analysis is depicted in Fig. 3(c), which shows a good agreement between experiment and theory. From the fits to the LDOS we have extracted radiative decay rates $\Gamma_{\mathrm{rad}}^{\mathrm{hom}}=1.0 \mathrm{~ns}^{-1}, 0.88 \mathrm{~ns}^{-1}$, and $0.82 \mathrm{~ns}^{-1}$ and nonradiative decay rates $\Gamma_{\text {nrad }}=1.2 \mathrm{~ns}^{-1}$, $1.0 \mathrm{~ns}^{-1}$, and $0.79 \mathrm{~ns}^{-1}$ for $\lambda_{c}=920 \mathrm{~nm}, 930 \mathrm{~nm}$, and 940 $\mathrm{nm}$, respectively. After averaging these values over wavelength and taking the experimental errors into account we obtain a $\mathrm{QE}=\Gamma_{\mathrm{rad}}^{\mathrm{hom}} /\left(\Gamma_{\mathrm{nrad}}+\Gamma_{\mathrm{rad}}^{\mathrm{hom}}\right)=(47 \pm 14) \%$ which is somewhat lower than values in excess of $90 \%$ reported for self-assembled QDs. ${ }^{18}$ The averaged radiative decay rate $\Gamma_{\mathrm{rad}}^{\mathrm{hom}}(\omega)=(0.9 \pm 0.2) \mathrm{ns}^{-1}$ allows us furthermore to determine an averaged OS $=10.1 \pm 2.6$ for the SCQDs. ${ }^{18}$ This result compares well with values on the order of 10 obtained for standard InAs QDs and is supported by diamagnetic coefficients below $10 \mu \mathrm{eV} / \mathrm{T}^{2}$ for SCQDs which is typical for small diameter QDs. ${ }^{22}$

We attribute the higher nonradiative decay rates for SCQDs mainly to tunneling and subsequent fast relaxation of carriers at defects close to the etched nanohole interface $18 \mathrm{~nm}$ below the QD layer (cf. Fig. 1). We have calculated the tunneling rate between the SCQDs and the nearby nanohole interface using the simulation tool NEXTNANO (www.nextnano.de) in order to test the validity of this interpretation. The corresponding tunneling rate through the 18 $\mathrm{nm}$ thick GaAs/ $\mathrm{Al}_{0.34} \mathrm{Ga}_{0.66} \mathrm{As}$ barrier (see above for the layer composition $)$ is given by $\Gamma_{\text {tun, }, / h}=\left(2 E_{0, e / h} / h\right) T_{e / h}$, where $E_{0, e / h}$ represents the energy of the electron (e) and hole (h) ground state in the QD. For a rough estimate of $\Gamma_{\text {tun, } e / h}$ we calculated the tunneling probability $T_{e / h}$ of electrons and holes under flat-band condition. While the corresponding tunneling rate of the holes $\left(\Gamma_{\operatorname{tun}, h}\right)$ is negligibly small, we obtain $\Gamma_{\text {tun }, e}=0.1 \mathrm{~ns}^{-1}$ for the electrons with $E_{0}=0.21 \mathrm{eV}$ and an ionization energy of $60 \mathrm{meV}$. The deviation from the experimental data $\left(\Gamma_{\text {nrad }} \approx 1 \mathrm{~ns}^{-1}\right)$ is attributed to Fermi level pinning at the nanohole interface and the related band bending. In fact, when varying the band bending, $\Gamma_{\text {tun,e }}$ $=1.0 \mathrm{~ns}^{-1}$ is obtained for a reasonably strong electrical field $E=30 \mathrm{kV} / \mathrm{cm}$ present in the growth direction. It is interesting to note that increasing the $\mathrm{Al}$ content in the $3 \mathrm{~nm}$ thick AlGaAs section from $34 \%$ to $74 \%$ would result in a more than two orders of magnitude lower tunneling probability, which could strongly enhance the QE of the SCQDs. Thus, further optimization in terms of the QE could focus on increasing the barrier height or thickness of the AlGaAs section in the spacer layer. We would like to point out that an exact description of the nonradiative decay process requires a detailed knowledge of the nature and the density of defects at the etched nanohole interface which is beyond the scope of this paper.

In summary we have performed detailed studies on the optical quality of site-controlled InAs QDs. By applying time-resolved spectroscopy on samples with varying thickness of the capping layer we measured the homogeneous medium radiative and nonradiative decay rates, from which we directly determined the QE and OS. The relatively high $\mathrm{QE}=(47 \pm 14) \%$ of the SCQDs is very promising for applications in the $C Q E D$ regime because the radiative decay rate and thus the QE can be enhanced by the Purcell effect, which is particularly feasible due to the accurate position control of the SCQDs. Furthermore, the present method of determining the $\mathrm{QE}$ and $\mathrm{OS}$ will be highly beneficial for the ongoing optimization of SCQDs.

This work was financially supported by the German Ministry of Education and Research project "QPENS" the European Commission (project "QPhoton") and the DFG via the strategic Japanese-German cooperative program project "Single quantum dot based lasers." Technical assistance by M. Emmerling and T. Steinl is gratefully acknowledged. S. Stobbe and P. Lodahl acknowledge financial support from the Danish Council for Independent Research (Technology and Production Sciences and Natural Sciences) and would like to thank P. T. Kristensen for valuable input to the modeling.

${ }^{1}$ J. P. Reithmaier, G. Sek, A. Löffler, C. Hofmann, S. Kuhn, S. Reitzenstein, L. V. Keldysh, V. D. Kulakovskii, T. L. Reinecke, and A. Forchel, Nature (London) 432, 197 (2004).

${ }^{2}$ T. Yoshie, A. Scherer, J. Hendrickson, G. Khitrova, H. M. Gibbs, G. Rupper, C. Ell, O. B. Shchekin, and D. G. Deppe, Nature (London) 432, 200 (2004).

${ }^{3}$ P. Michler, A. Kiraz, C. Becher, W. V. Schoenfeld, P. M. Petroff, L. Zhang, E. Hu, and A. Imamogl ǔ, Science 290, 2282 (2000).

${ }^{4}$ S. Strauf, K. Hennessy, M. T. Rakher, Y.-S. Choi, A. Badolato, L. C. Andreani, E. L. Hu, and P. M. Petroff, Phys. Rev. Lett. 96, 127404 (2006)

${ }^{5}$ S. Reitzenstein, T. Heindel, C. Kistner, A. Rahimi-Iman, C. Schneider, S. Höfling, and A. Forchel, Appl. Phys. Lett. 93, 061104 (2008).

${ }^{6}$ Y. Nakamura, O. G. Schmidt, N. Y. Jin-Phillipp, S. Kiravittaya, C. Muller, K. Eberl, H. Grabeldinger, and H. Schweizer, J. Cryst. Growth 242, 339 (2002).

${ }^{7}$ M. H. Baier, S. Watanabe, E. Pelucchi, and E. Kapon, Appl. Phys. Lett. 84, 1943 (2004).

${ }^{8}$ Z. Song, T. Usuki, S. Hirose, K. Takemoto, Y. N. N. Yokoyama, and Y. Sakuma, Appl. Phys. Lett. 86, 113118 (2005).

${ }^{9}$ O. G. Schmidt, Lateral Alignment of Epitaxial Quantum Dots (Springer, Berlin, Heidelberg, 2007)

${ }^{10}$ C. Schneider, M. Strauß, T. Sünner, A. Huggenberger, D. Wiener, S. Reitzenstein, M. Kamp, S. Höfling, and A. Forchel, Appl. Phys. Lett. 92 183101 (2008).

${ }^{11}$ T. Sünner, C. Schneider, M. Strauß, A. Huggenberger, D. Wiener, S. Höfling, M. Kamp, and A. Forchel, Opt. Lett. 33, 1759 (2008).

${ }^{12}$ P. Gallo, M. Felici, B. Dwir, K. A. Atlasov, K. F. Karlsson, A. Rudra, A Mohan, G. Biasiol, L. Sorba, and E. Kapon, Appl. Phys. Lett. 92, 263101 (2008).

${ }^{13}$ C. Schneider, T. Heindel, A. Huggenberger, P. Weinmann, C. Kistner, M. Kamp, S. Reitzenstein, S. Höfling, and A. Forchel, Appl. Phys. Lett. 94, 111111 (2009)

${ }^{14}$ C. Schneider, A. Huggenberger, T. Sünner, T. Heindel, M. Strauss, S Göpfert, P. Weinmann, S. Reitzenstein, L. Worschech, M. Kamp, S. Höfling, and A. Forchel, Nanotechnology 20, 434012 (2009).

${ }^{15}$ P. Atkinson, O. G. Schmidt, P. S. Bremner, and D. A. Ritchie, C. R. Phys 9, 788 (2008)

${ }^{16}$ S. Kiravittaya, M. Benyoucef, R. Zapf-Gottwick, A. Rastelli, and O. G Schmidt, Appl. Phys. Lett. 89, 233102 (2006)

${ }^{17}$ T. Tran, A. Muller, C. K. Shih, P. S. Wong, G. Balakrishnan, N. Nuntawong, J. Tatebayashi, and D. L. Huffaker, Appl. Phys. Lett. 91, 133104 (2007).

${ }^{18}$ J. Johansen, S. Stobbe, I. S. Nikolaev, T. Lund-Hansen, P. T. Kristensen, J. M. Hvam, W. L. Vos, and P. Lodahl, Phys. Rev. B 77, 073303 (2008).

${ }^{19}$ S. Stobbe, J. Johansen, P. T. Kristensen, J. M. Hvam, and P. Lodahl, Phys. Rev. B 80, 155307 (2009).

${ }^{20}$ H. Drexhage, J. Lumin. 1-2, 693 (1970).

${ }^{21}$ M. Bayer and A. Forchel, Phys. Rev. B 65, 041308 (2002)

${ }^{22}$ S. Reitzenstein, C. Schneider, S. Münch, C. Kistner, M. Strauss, A. Huggenberger, P. Franeck, P. Weinmann, M. Kamp, S. Höfling, L. Worschech, and A. Forchel, Acta Phys. Pol. A 116, 445 (2009). 